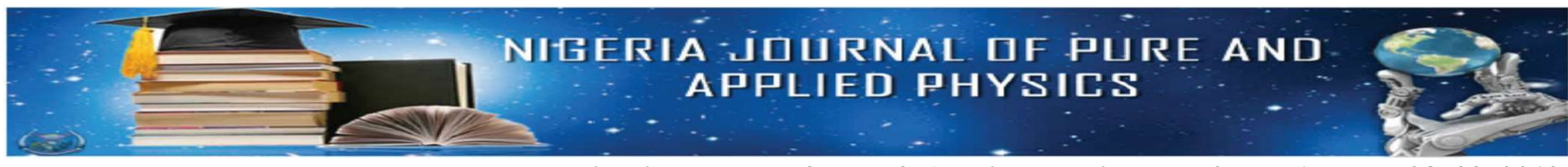

Nigeria Journal of Pure \& Applied Physics, Vol. 9, No. 1, pages 28-33, 2019

\title{
Chaotic assessment of the key performance indicators for a GSM Network congestion in an election period in Nigeria
}

\author{
A. O. Adelakun ${ }^{1, \dagger}$, B. Y. Lawal ${ }^{2}$, M. A. Adekoya ${ }^{1,3}$, K. E. Ukhurebo ${ }^{3}$ \\ ${ }^{1}$ Department of Physics, Federal University of Technology, Akure, Nigeria. \\ ${ }^{2}$ Department of Physics, University of Africa, Toru-orua, Bayelsa State, Nigeria. \\ ${ }^{3}$ Department of Physics, Edo University, Iyamho, Edo State, Nigeria. \\ †Corresponding Author's Email: aoadelakun@futa.edu.ng
}

\begin{abstract}
This paper investigates the chaos-based assessment of the Key performance Indicators (KPIs) for the Cellular network congestion during and after 2011 People Democratic Party (PDP) presidential primary election in Eagle Square within Abuja and its environs. Chaotic quantifiers such as average mutual information, Lyapunov exponents and specific attenuation were employed to study the congestion rate or dynamical state of the network. The chaotic assessment of the Wideband Code Division Multiplexing Access (WCDMA) data services provided by MTN network using KPIs are reported. The data retrieved from the three Base Transceiver Stations (BTSs) were used to analyse the performance of the network. The results revealed the state of dynamical behaviour of the congestion during the study period.
\end{abstract}

Keywords: Key performance Indicators (KPIs); Wideband Code Division Multiplexing Access (WCDMA); Global System of Mobile communication (GSM); Base Transceiver Station (BTS); Chaotic Quantifiers

\section{INTRODUCTION}

GSM Congestion is reduction in the quality of network service when the link carries more than its carrying capacity. When blocking of network occurs, no free path can be provided for an offered call [1] i.e. when a subscriber cannot obtain a connection to the wanted subscriber immediately. Kuboye, 2016 indicated the reasons why a call could not be completed such as Pulse Code Modulation Congestion (PCMC), Dedicated Control Channel Congestion (DCHC), Traffic Channels Congestion (TCHC), Common Control Channels Congestion (CCCHC) and too many users on the net-work to mention a few [2-4]. However, a resolution for the congestion problem in Public land mobile communication network is to predict the long-term forecasting for a whole traffic for both programming of GSM network capacity and dynamic optimization of access network. Revolution in the Quality of service of the Network provider in terms of retainability, accessibility and voice quality in recent years $[5,6]$ have opened door in the area of Telecommunication such as security alert, secured communication, e-learning, mobile banking, short message services e.t.c. Quality of Service (QoS) is an important key performance indicator (KPI) that is used in determining the efficiency of an industry in terms of services rendered [7]. Other large number of factors in the environment also affect the signal that is transmitted by the transmitting antenna (from Base Transceiver System to Mobile Station) and received by the receiving antenna (by Mobile Station from Base Transceiver System). Quality of network can be improved by eliminating the factors that affects the KPIs performance, both from external and internal sources. High speed data, qualitative voice calls and other usefull applications such as fast internet connections are ingredient for successful call and optimization of network services. Global System Mobile Communication (GSM) services have been transformed from $1 \mathrm{G}, 2 \mathrm{G}, 3 \mathrm{G}$ and $4 \mathrm{G}$ due to good coverage, capacity and quality of the network. As the number of services and subscribers increase in Nigeria, the demand for good QoS has become an issue. GSM is the best means of voice telephony employed globally. It is a collection of a number of local base stations like building blocks to form a giant network. A Base Transceiver Station (BTS) contains an equipment for transmitting and receiving radio signals and equipment for encrypting and decrypting information with base station controller. Typically, BTS interconnect a number of mobile users to the next nearest mobile station of a particular network service provider [8]. Generally, the overall performance of a particular network having number of BTS is based on the individual performance of the BTSs. In order for the end users to have the best experience and derive optimum satisfaction from the services, there is need for proper monitoring, control and evaluation [9-12]. Network management requires real-time monitoring of the current network status, performance and the ability to take prompt action on the network when necessary. It should be noted that the complete range of network status and performance parameters are not necessary for the introduction of a net-work management capability. Performance and quality of service (QoS) evaluation are the most important to mobile operators as the revenue generation and customer satisfaction are directly related to network performance and quality. Mkheimer and Jamoos (2012), used two approaches to evaluate the network 
performance namely: key performance indicators (KPIs) and drive test. They suggested that the average $\mathrm{TCH}$ of about $0.76 \%$ in November 2010 to $0.62 \%$ in October 2011 are dropped. In addition, about $7.76 \%$ of the collected samples from the drive test lie in level 4 which is the worst level in terms of signal quality and signal strength. Moreover, only $65.5 \%$ of the collected samples lie in level 1 which is the best level in terms of signal quality and strength [13]. In Nigeria, the telecommunication industry is rapidly growing and many operators and their operations put forth different services, but in the diverse services provided, most of the subscribers do not receive satisfaction due to the poor nature of services available on these networks. Competition in the industry is a likely tool that would indirectly improve services rendered, but this is not yet the case in Nigeria since competition in the telecommunications industry is fairly new and may be described as being inefficient. In this work, the main focus is to report the chaotic or congestion dynamics of the reported KPIs during and after 2011 PDP presidential primary election in Eagle Square Abuja and its environs.

\section{KPIS MODELS}

KPIs are network statistical indicators that can be used for assessing the performance and Quality of service of GSM Network Service Providers. These indicators vary temporally and spatially due to variation in the capacity and configuration of each cell and the number of active users locking to each cell in a given frame. Therefore, it is wrong to generalize the QoS of a network for a large number of cells covering an entire state or country. Network operation status can be understood with regard to the traffic volume on the network, as well as providing statistical data to understand network performance [13]. KPIs are commonly used to characterize the performance of a network operator for a sizeable land mass such as community, village, city, local government area e.t.c where only one Base station controls the activity of a number of BTSs (cells). KPI are dynamical variables whose values can be influenced by a number of factors, hence, there is need for monitoring, control and analysis of these variables. The major KPIs investigated in this work are Call Setup Success Rate (CSSR), Hand Over Success Rate (HOSR), Traffic Channel Congestion Rate (TCH-CR) or percentage of congestion (PCONG), percentage Drop Call Rate (PDROP), Control Channel Set-up Failure (CCSF) or percentage rate of failure (PCTRFAIL) and traffic strength (ERLANG). Figure 1 capture the three BST covering time series analysis for the proposed KPIs for the six days between January 10 to 15 , 2011. KPIs are commonly used to characterize the performance of a network operator for a sizeable land mass such as community, village, city, local government area e.t.c where only one Base station controls the activity of a number of BTSs (cells). KPI are dynamical variables whose values can be influenced by a number of factors, hence, there is need for monitoring, control and analysis of these variables. The major KPIs investigated in this work are Call Setup Success Rate (CSSR), Hand Over Success Rate (HOSR),
Traffic Channel Congestion Rate (TCH-CR) or percentage of congestion (PCONG), percentage Drop Call Rate (PDROP), Control Channel Set-up Failure (CCSF) or percentage rate of failure (PCTRFAIL) and traffic strength (ERLANG). Figure 1 capture the three BST covering time series analysis for the proposed KPIs for the six days between January 10 to $15,2011$.

(1) Call Setup Success Rate (CSSR) also referred to as Standalone Dedicated Control Channel Success Rate (SDCCHSR) or service availability is a measure of the number of calls that are successfully set-up or connected through the overall length of time required to establish a call between users.

(2) Hand Over Success Rate (HOSR): This is the ability of live calls (established channels) on a particular network core to be successfully transferred to another network core. When a mobile user who on live call travels through the coverage area of one cell to another, such call is expected to be successfully handed over to the next cell without interruption. For a given network within a particular period (usually 60 minutes), HOSR values describe the percentage of calls that were successfully handed over as mobile users from one cell to another.

(3) Percentage Traffic Channel Congestion Rate (TCH-CR PCONG) : Each BTS has a maximum permissible number of calls (known as Traffic Channel TCH) that can be set up at a time. When a subscriber locking to a particular BTS attempts to a make a call, the TCH in use must be less than the maximum $\mathrm{TCH}$; otherwise, bid to establish call will be rejected.

(4) Drop call Rate (PDROP): It is a measure of the rate at which live calls were prematurely terminated by network issues. It is commonly caused by poor HOSR, high traffic channel congestion rate (TCH-CR), interference, and frequency reuse, fluctuations in received signal strength or sudden network breakdown. Service providers are always conscious of PDROP status because an astronomical increase in PDROP is an indication of serious network problem; thus, requires urgent attention.

(5) PCTRFAIL or Control Channel Set-up Failure (CCSF): Every BTS has a specific maximum number of traffic channels that can be set up at a time. When a subscriber attempts to make a call, a Dual Tone MultiFrequency (DTMF) signal must be returned to the potential caller indicating there is or are free channels available to begin the phone call process at the moment otherwise no call can be initiated. The percentage of unsuccessful control channel set up in a given period (usually 60 mins) is referred to as CCSF.

(6) ERLANG: It is a special unit used for statistical measure of the voice traffic den-sity in a telecommunications system and it is widely used because, for any element in a telecommunications system, whether it is a landline, or uses cellular technology, it is necessary to be able to understand the traffic volume. Erlang plays an important role in the design of GSM network It enables network designers to predict the traffic volume when designing network coverage for voice telephony. 


\section{OPTIMIZATION PROCESSES}

For the fact that primary Election Day usually involve massive political party members from all the 36 states of the federation, hence a heavy traffic is ascertained within the FCT and the environs. To this effect, the steps involved in optimization of traffic or congestion of network consist of two parts, namely: neighbour relation plan and capacityinterference analysis [13]. The capacity and interference analysis involve determination of peak carried traffic for each cell of the base stations. Figure 1 shows the KPIs time series for the three studied Cells. The traffic intensity offered by each user $(\mathrm{Au})$ which is a measure of the utilization or the average channel occupancy measured in units of ERLANG [14] is given by

$$
\mathrm{A}_{\mathrm{U}}=\lambda \mathrm{H}
$$

where $\lambda$ is the average number of call requests per unit time and $\mathrm{H}$ is the average duration of a call. For instance, a mobile system with capacity of $U$ users will have the total offered traffic intensity (A) is defined as

$$
\mathrm{A}=\mathrm{U} \mathrm{A}_{\mathrm{U}}
$$

The signal-to-interference $(\mathrm{S} / \mathrm{I})$ ratio of a mobile receiver which monitors a forward control channel [14] can be approximated as

$$
\frac{S}{I}=\frac{Q^{n}}{i_{0}}=\frac{(3 N)^{n}}{i_{0}}
$$

where $\mathrm{Q}$ is the co-channel reuse ratio, $\mathrm{n}$ is the path loss exponent in which it depends on the specific wireless propagation of the environment, $\mathrm{i}_{O}$ is the number of first layer co-channel interfering cells while $\mathrm{N}$ is the cluster size.

The Quality of service can be improved by increasing the co-channel reuse ratio, $\mathrm{S} / \mathrm{I}$ or the number of control channels. In addition, the ability of a user to access the system during the busiest or peak hours i.e. grade of service of a mobile system which can be denoted as the probability of a blocked call rate [14] can be described as follows:

$$
G o S=\frac{A^{C}}{C !} \sum_{k=o}^{C}\left(\frac{k !}{A^{k}}\right)
$$

where $\mathrm{A}$ is the total traffic intensity and $\mathrm{C}$ denote the total number of TCHs in a cell [11].

\section{METHODOLOGY AND DATA ANALYSIS}

\subsection{Data Collection}

Based on the largest number of mobile subscribers as at June 2016 in the country [15], the traffic GSM service provider (MTN) data for the three BTSs covering Eagle Square and its environment located within the Federal Capital Territory (FCT) of Nigeria were obtained through the Base Station Controller (BSC) which serves as the parent base station for these BTSs. The traffic data span from 10th of January to 15th of January 2011. The justification for the traffic period employed was to study and analyse the congestion of the
GSM voice service before (10th, 11th and 12th), during (13th) and after (14th and 15th) Peoples Democratic Party (PDP) presidential primary election in 2011.

\subsection{Chaotic Dynamics Analysis}

In nonlinear science, system can either be periodic, quasiperiodic, chaotic or hyper-chaotic depending on the state of the system. In order to test for degree of chaoticity of the Cellular Network Congestion during or after the presidential primary election, chaotic quantifiers such as Lyapunov exponent, average mutual information to time delay and specific attenuation must be investigated. Also, there is need to identify the state of congestion which leads to reduction in the quality of network. Lyapunov exponent as one of the major tools has been an important quantifier in the determination of chaos in time series, representing natural systems like the congestion in GSM network [16, 17]. Depending on the dimension of a dynamical system, a system can be periodic if one of the Lyapunov exponent is negative, quasi-periodic if the Lyapunov exponent is zero. In addition, a chaotic system can also occur if one of the Lyapunov exponent is positive and hyperchaotic if two of the Lyapunov exponents is positive. The largest Lyapunov exponent $\lambda_{1}$ can be used to determine the rate of divergence [18-20] as:

$$
\lambda_{1}=\frac{1}{t} \ln \frac{\Delta x(t)}{x(0)}=\frac{1}{t} \sum_{i=1}^{t} \ln \frac{\Delta x\left(t_{i}\right)}{\Delta x\left(t_{i-1}\right)}
$$

where $t$ is the sampling period of the time series and $\Delta x$ is the distance between the ith pair of nearest neighbors after discrete-time steps. The Maximum Lyapunov exponent was computed for each KPIs data for the three cells. For example, in Figure 2, the employed KPIs data clearly shows the chaoticity of the congestion, with positive maximum Lyapunov exponents covering the range $\leq 100$ hours for PDROP and HOSR respectively for the three base stations, while quasi-periodic within the range $>100$ which depicts low congestion periods during the election. That is, more congestion of network services occurs as we recorded different network services from different providers, which actually predicts the period of high and low congestion i.e. periods of network failure, for the chosen network service. In figure 3, the information for the average mutual information with respect to the time delay for the three cells were captured. The minimum marked for all the six KPIs can be considered as the optimal time delay. In cell A of Figure 3 (b), the PDROP is usually high when there is traffic congestion i.e. PCONG is the major determinant. Similarly, Figure 3(b) and (c) also depicts the divergence of the trajectory. The specific attenuation against the BTS 


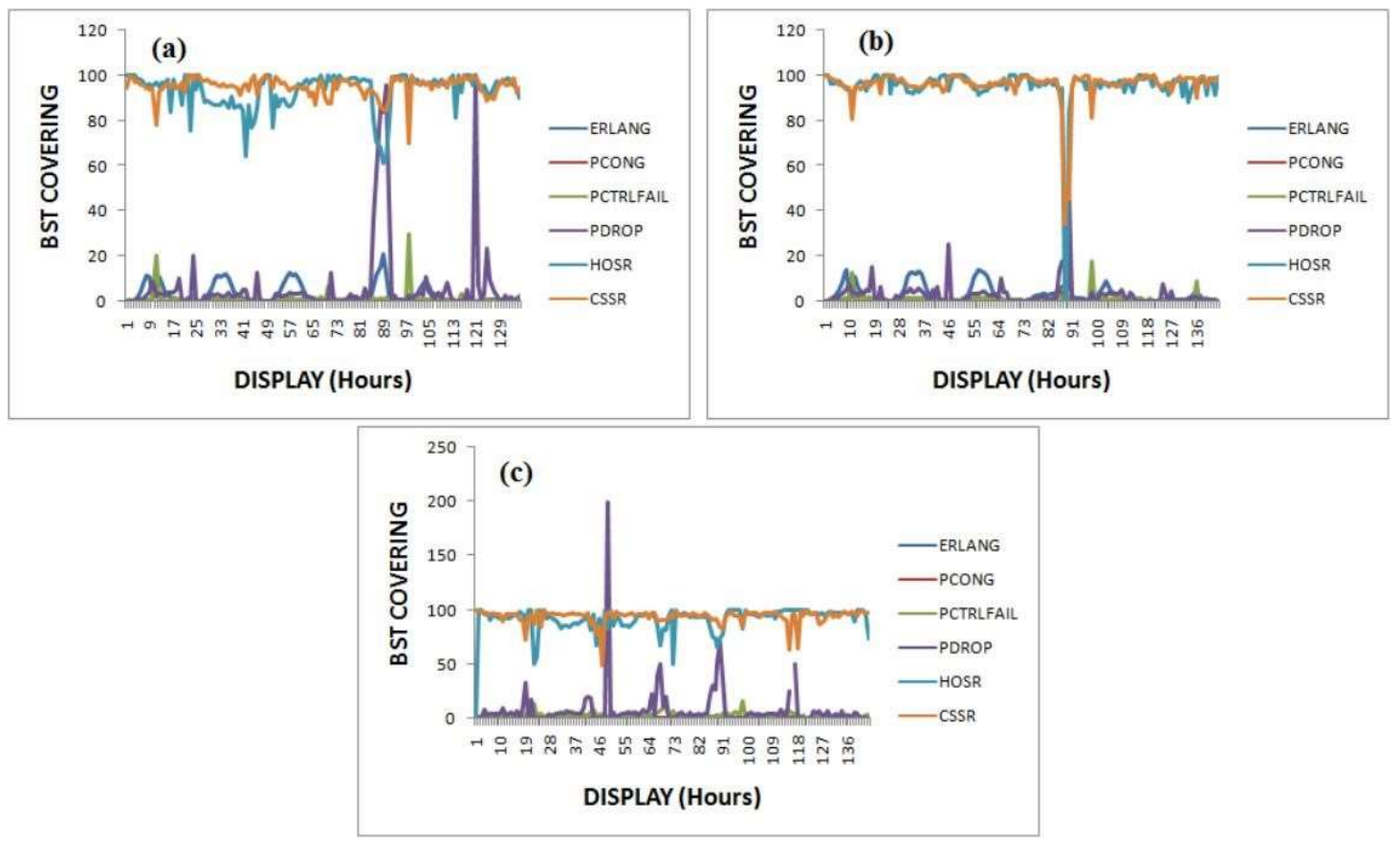

Fig. 1. BST covering for the six KPIs (a) cell A, (b) cell B and (c) cell C
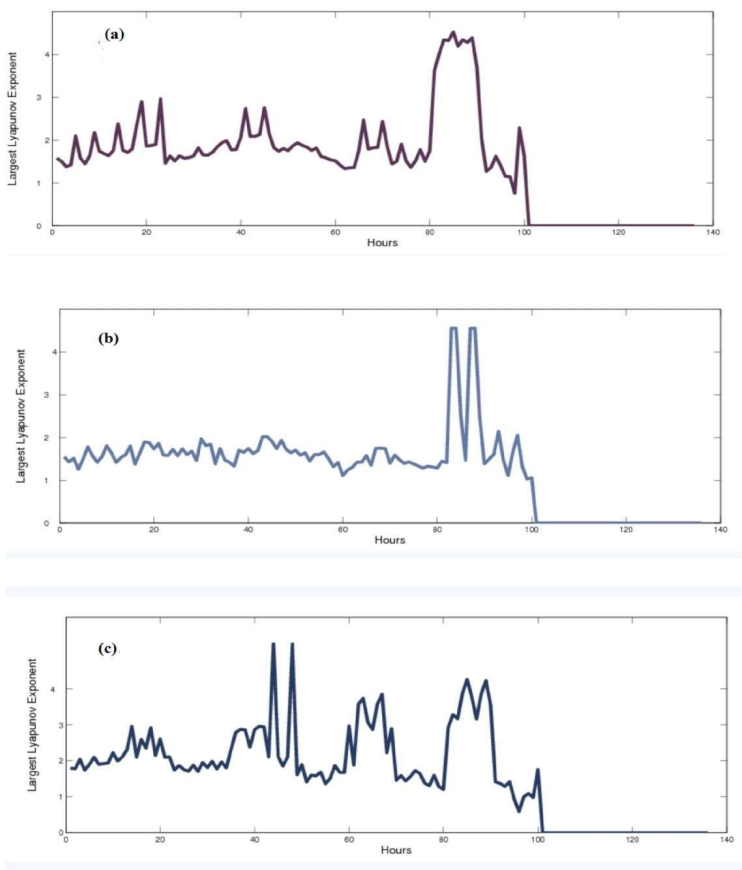

Fig. 2. Maximum Lyapunov exponents for (a) cell A (PDROP), (b) cell B (HOSR) and (c) cell C (PDROP). covering area for the three Cells were also plotted as shown in Figure 4.

Attenuation is a loss of signal strength measured in $\mathrm{dB}$ that reduces a connections maximum speed available due to the need for multiple repeat transmissions. The causes of attenuation may be noise, physical surroundings and travel distance. And any or all of these causes can actually affect the strength of the signal i.e. As attenuation increases, the more distorted and unintelligible the transmission (e.g. a phone call or email you are trying to send) becomes. The Hand over success rate (HOSR) also referred to as Inter-Cell link rate is one of the major KPIs that relate directly with service availability. With high percentage of attenuation discovered in cell $\mathrm{A}, \mathrm{B}$ and $\mathrm{C}$, we also observed an interruption in Cell $\mathrm{C}$ which occurred during 90 hours due to abrupt loss of signal. In Figure 4, high attenuation occurred at $20 \leq$ Hours $\leq 90$ for HOSR, with peaks of about $650 \mathrm{~dB} / \mathrm{km}$ at 25 and 75 hours for Cell $\mathrm{C}$ respectively. IS THIS NECESSARY?

\section{CONCLUSION}

In this paper, the chaotic characteristic analyses of the Key performance Indicators (KPIs) for the Cellular network congestion during and after 2011 PDP presidential primary election in Eagle Square Abuja and its environs have been reported. Chaotic quantifiers such as average mutual information, Lyapunov exponents and specific attenuation (SA) have been used to study the dynamical state of the Cellular network conges-tion. The confirmation of chaoticity for the choosen KPIs have been established with 


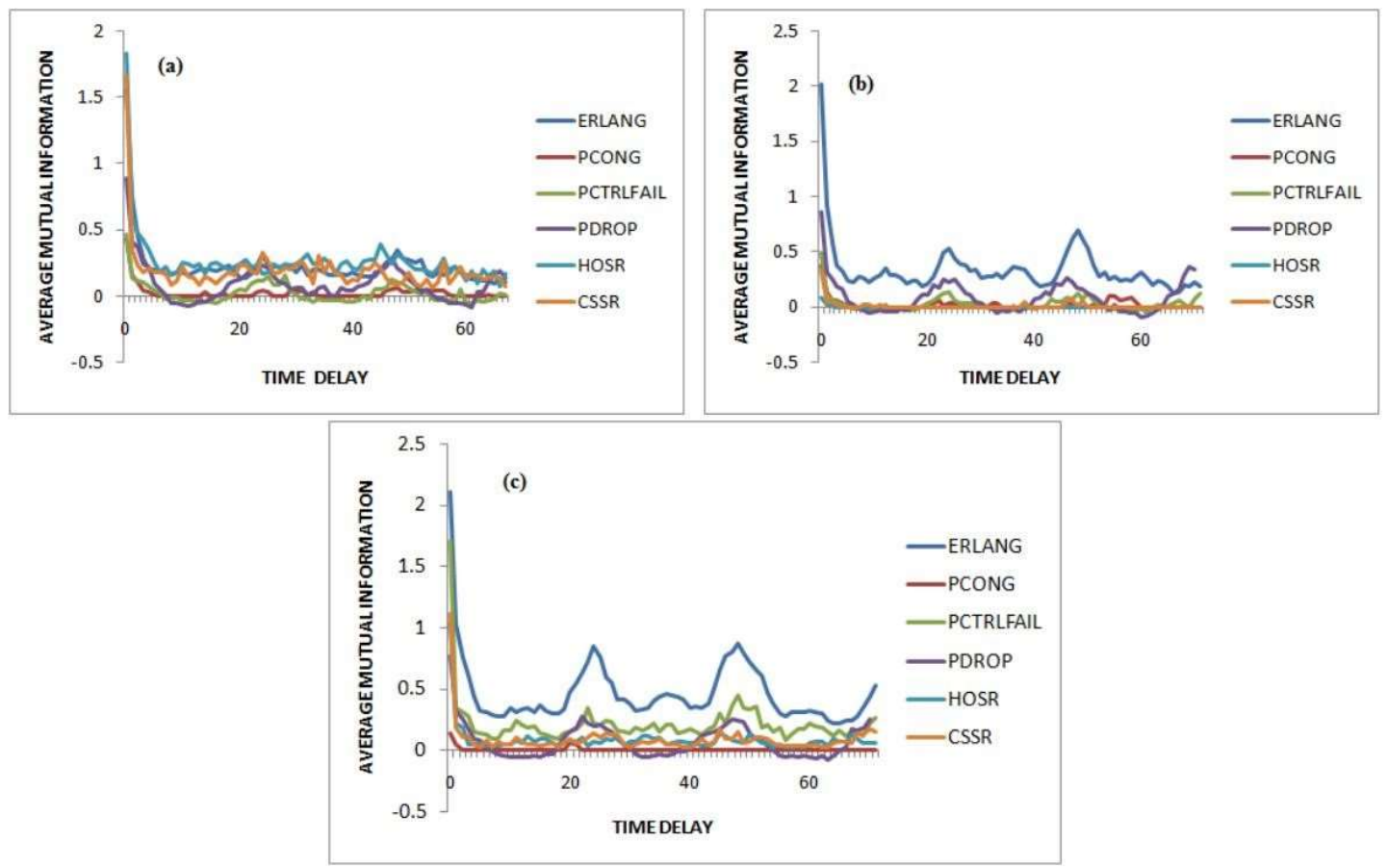

Fig. 3. Average Mutual information for all KPIs against the time delay for (a) cell A, (b) cell B and (c) cell C.

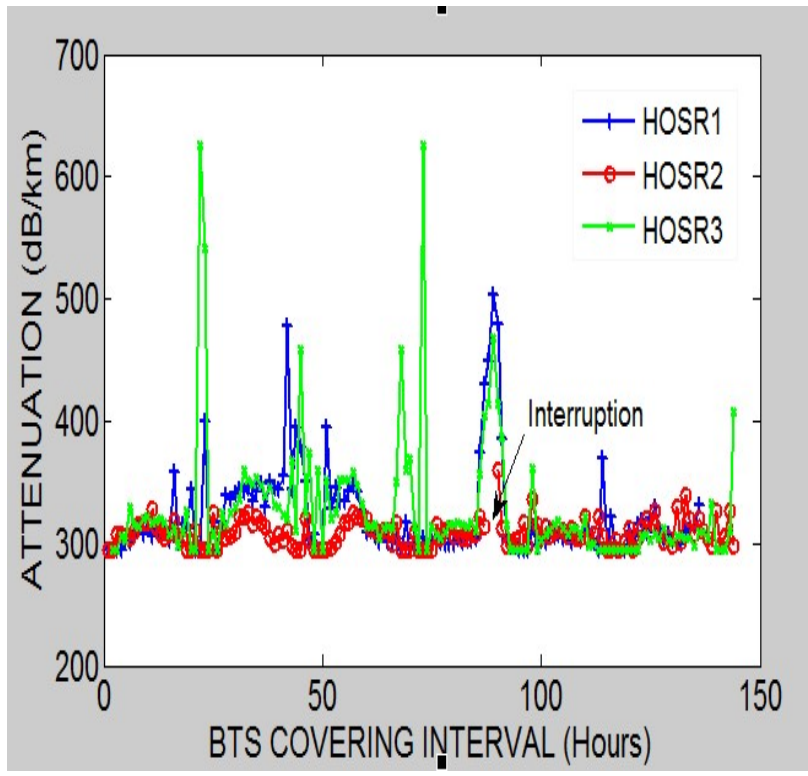

Fig. 4. Specific Attenuation for HOSR in cell A, cell B and cell C

chaotic regions and quasi-periodic states which emanate from the cellular network congestion. Finally, the chaotic quantifiers used in this research work has established the hours with high congestion and low congestion of network services which may leads to failure of network during the study period.p

\section{REFERENCES}

[1.] Harte L, Levine R, and Livingston G. (1999). "GSM Superphones". McGraw Hill 71: 45-47.

[2.] Kuboye B.M. (2010). "Optimization models for minimizing congestion in Global System for Mobile Communications (GSM) in Nigeria". Journal Media and Communication Studies 2(5): 122-126.

[3.] Mehrotra A. (1997). "GSM System Engineering". Artech house, Inc., Norwood MA 70-73, USA. ISBN:0890068607.

[4.] Syski R. (1986). "Introduction to Congestion Theory in Telephone Systems". Elserier Science Publishers B.V.

[5.] Kyriazakos S, Gkroustiotis V, Karambalis C, Kechagias C, Papaoulakis N, Nikitopoulos D. and Karetsos, G. (2002). "Performance evaluation of GSM and GPRS systems based on measurement campaigns". Proceedings of the 5th IEEE International Symposium on Wireless Personal Multimedia Communications 3:1167-1171

[6.] Adegoke A.S, and Babalola I.T. (2011). "Quality of service analysis of GSM tele-phone system in Nigeria". American Journal of Scientific and Industrial Research 2(5): 707-712. doi:10.5251/ajsir.2011.2.5.707.712.

[7.] Osunade O, and Oyesanya O.O. (2016). "Independent Quality of Service (QoS) Validation of A Telecommunication Provider in South-West Nigeria". Inter. Jour. of Computer and Information Technology 5(1): 119124.

[8.] Kollar M. (2008). "Evaluation of real call set up success rate in GSM". Acta Electrotechnica et Informatica 8(3):5356. 
[9.] Haider B, Zafuaalh M, and Islam M.K. (2009). "Radio frequency optimization and QoS evaluation in operational GSM network". Proceedings of the World Congress on Engineering and Computer Science 1: 393-398, San Francisco, USA.

[10.] Mishra A.R. (2004). "Fundamentals of Cellular Network Planning and Optimization 2G/2.5G/3G Evolution to 4 G'. Wiley and Sons, Ltd., England.

[11.] Mishra A.R. (2007). "Advanced Cellular Networks Planning and Optimization 2G/2.5G/3G Evolution to 4G"., Wiley and Sons, Ltd., England.

[12.] Idigo V.E, Azubogu A.C.O, Ohaneme C.O, and Akpado K.A. (2012). "Real-Time Assessments of QoS of Mobile Cellular Networks in Nigeria”. Inter. Jour. of Engineering Inventions 1(6): 64-68.

[13.] Mkheimer B, and Jamoos A. (2012). "Evaluation and optimisation of GSM network in Jenien City, Palestine". Int. J. Mobile Network Design and Innovation 4(4): 201-213.

[14.] Lawal B.Y., Ukhurebor K.E., Adekoya M.A., Aigbe E.E. (2016). "Quality of Service and Performance Analysis of A GSM Network in Eagle Square, Abuja and Its Environs, Nigeria", Int. Jour. of Scientific and Engineering Research 7(8): 1992-1999.
[15.] Rappaport, T.S. (2001)." Wireless Communications: Principles and Practice", Prentice Hall, USA.

[16.] Unnikrishnan, K. (2008)." Comparism of Chaotic aspect of Magnetosphere under various physical conditions using E Index time series”. Ann. Geophys. 26:941-953.

DOI: 1.5194/angeo-26-941.

[17.] nnikrishnan, K. (2010). "A comparative study on Chaoticty of Equitorial/Low lattitude ionosphere over Indian subcontinent during Geomagnetically quiet and disturbed period", Nonlin. Processes Geophys. 17:765-776. DOI: 10.5194/npg-17-765-2010.

[18.] Wolf, A., Swift, J. B., Swinney, H. L., and Vastano, J. A. (1985). "Determining Lyapunov exponents from a time series", Physica D., 16, 285-317.

[19] Adelakun A.O., Ojo J.S., Edward O.V. (2019). "Quantitative Analyses of Complexity and Nonlinear trend of Radio Refractivity Gradient in the troposphere", Advances in Space Research, In press

https://doi.org/10.1016/j.asr.2019.09.055.

[20] Ojo J.S., Adelakun A.O., Edward O.V. (2019). "Comparative study on Radio Refractivity Gradient in the troposphere using Chaotic Quantifiers", Heliyon 5, https://doi.org/10.1016/j.heliyon.2019.e02083 